\title{
Optical and acoustic sensing using Fano-like resonances in dual phononic and photonic crystal plate
}

Amoudache, Samira; Moiseyenko, Rayisa; Pennec, Yan; Rouhani, Bahram Djafari; Khater, Antoine; Lucklum, Ralf; Tigrine, Rachid

Published in:

Journal of Applied Physics

Link to article, DOI:

$10.1063 / 1.4944600$

Publication date:

2016

Document Version

Publisher's PDF, also known as Version of record

Link back to DTU Orbit

Citation (APA):

Amoudache, S., Moiseyenko, R., Pennec, Y., Rouhani, B. D., Khater, A., Lucklum, R., \& Tigrine, R. (2016).

Optical and acoustic sensing using Fano-like resonances in dual phononic and photonic crystal plate. Journal of Applied Physics, 119(11), [114502]. https://doi.org/10.1063/1.4944600

\section{General rights}

Copyright and moral rights for the publications made accessible in the public portal are retained by the authors and/or other copyright owners and it is a condition of accessing publications that users recognise and abide by the legal requirements associated with these rights.

- Users may download and print one copy of any publication from the public portal for the purpose of private study or research.

- You may not further distribute the material or use it for any profit-making activity or commercial gain

- You may freely distribute the URL identifying the publication in the public portal 


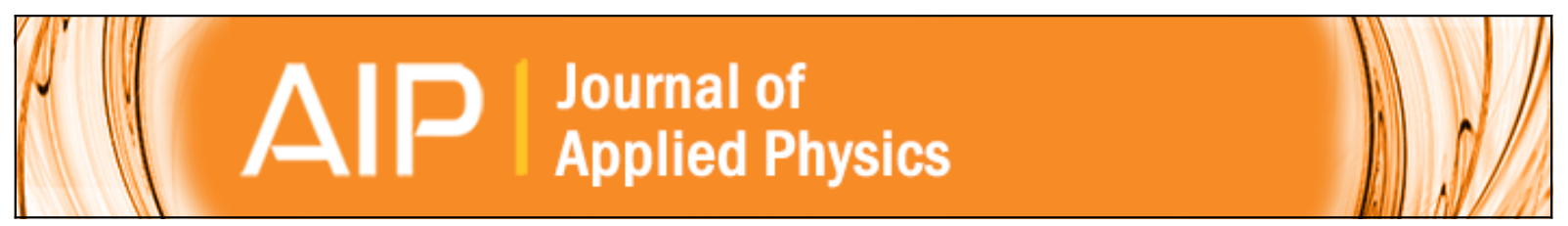

\section{Optical and acoustic sensing using Fano-like resonances in dual phononic and photonic crystal plate}

Samira Amoudache, Rayisa Moiseyenko, Yan Pennec, Bahram Djafari Rouhani, Antoine Khater, Ralf Lucklum, and Rachid Tigrine

Citation: Journal of Applied Physics 119, 114502 (2016); doi: 10.1063/1.4944600

View online: http://dx.doi.org/10.1063/1.4944600

View Table of Contents: http://scitation.aip.org/content/aip/journal/jap/119/11?ver=pdfcov

Published by the AIP Publishing

\section{Articles you may be interested in}

Refractive index dispersion sensing using an array of photonic crystal resonant reflectors

Appl. Phys. Lett. 107, 061101 (2015); 10.1063/1.4928548

Simultaneous sensing of light and sound velocities of fluids in a two-dimensional phoXonic crystal with defects

J. Appl. Phys. 115, 134503 (2014); 10.1063/1.4870861

Design of acoustic beam aperture modifier using gradient-index phononic crystals

J. Appl. Phys. 111, 123510 (2012); 10.1063/1.4729803

Sub-wavelength phononic crystal liquid sensor

J. Appl. Phys. 110, 026101 (2011); 10.1063/1.3610391

APL Photonics

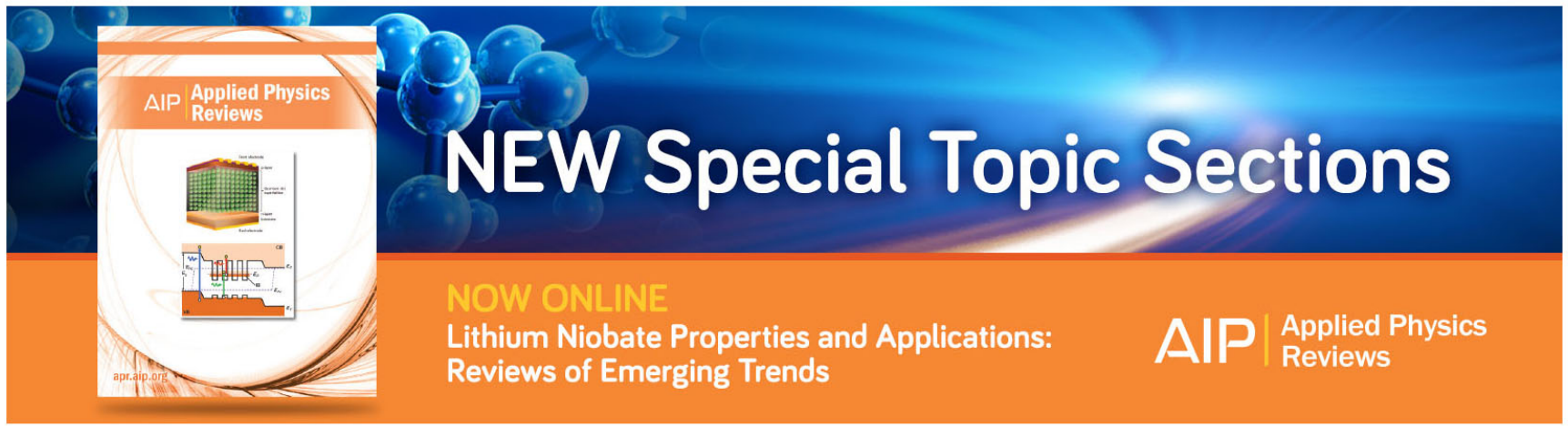




\title{
Optical and acoustic sensing using Fano-like resonances in dual phononic and photonic crystal plate
}

\author{
Samira Amoudache, ${ }^{1,2}$ Rayisa Moiseyenko, ${ }^{3}$ Yan Pennec, ${ }^{1, a)}$ Bahram Djafari Rouhani, ${ }^{1}$ \\ Antoine Khater, ${ }^{4}$ Ralf Lucklum, ${ }^{5}$ and Rachid Tigrine ${ }^{2}$ \\ ${ }_{1}^{1}$ Institut d'Electronique, de Microélectronique et de Nanotechnologie, Université de Lille 1, 59655 Villeneuve \\ d'Ascq, France \\ ${ }^{2}$ Laboratoire de Physique et Chimie Quantique, Université Mouloud Mammeri, B.P. 17 RP, 15000 Tizi-Ouzou, \\ Algeria \\ ${ }^{3}$ Department of Physics, Technical University of Denmark, DTU Physics, Building 309, DK-2800 Kongens \\ Lyngby, Denmark \\ ${ }^{4}$ Institut des Molécules et Matériaux du Mans (IMMM), UMR CNRS 6283, l'UNAM, Université du Maine, \\ 72085 Le Mans, France \\ ${ }^{5}$ Institute of Micro and Sensor Systems (IMOS), Otto-von-Guericke-University, P.O. Box 4120, \\ D-39016 Magdeburg, Germany
}

(Received 11 November 2015; accepted 8 March 2016; published online 21 March 2016)

We perform a theoretical study based on the transmissions of optical and acoustic waves normally impinging to a periodic perforated silicon plate when the embedded medium is a liquid and show the existence of Fano-like resonances in both cases. The signature of the resonances appears as well-defined asymmetric peaks in the phononic and photonic transmission spectra. We show that the origin of the Fano-like resonances is different with respect to the nature of the wave. In photonic, the origin comes from guided modes in the photonic plate while in phononic we show that it comes from the excitation of standing waves confined inside the cavity coming from the deformation of the water/silicon edges of the cylindrical inclusion. We finally use these features for sensing and show ultra-sensitivity to the light and sound velocities for different concentrations of analytes. (C) 2016 AIP Publishing LLC. [http://dx.doi.org/10.1063/1.4944600]

\section{INTRODUCTION}

The extraordinary optical transmission, known as EOT, has been introduced in 1998 by Ebbessen through a work based on a metallic film patterned with periodical cylindrical holes. ${ }^{1}$ Following this original work, many papers have discussed the physical origin of this effect ${ }^{2}$ considering the competition between three kinds of fundamental physical properties, namely, the Surface Plasmon Polariton (SPP) excitation, the Fabry Pérot resonances in the apertures, and the modes guided through the metallic crystal plate. All these effects can couple separately or together with the external incident radiation. Similarly, the demonstration and the description of Fano resonances in dielectric photonic crystal plates under normal illumination have been provided in terms of Bragg scattering and guided resonant plate modes. ${ }^{3-5} \mathrm{~A}$ few years later, this unexpected phenomenon has been demonstrated in phononic crystal, considering air-borne acoustic periodic structure under normal incidence. Papers have been devoted to onedimensional acoustic gratings with sub-wavelength slits ${ }^{6,7}$ or 2D periodic slabs drilled with air holes. ${ }^{8-10}$ Just a few works report on the reverse situation, i.e., when the embedded medium is solid and the interfacial membrane is made of air. This situation has been discussed in 2D structure with bulk elastic waves and in phononic membrane with incident Lamb waves. ${ }^{11,12}$ Khelif et al. investigated theoretically the propagation of elastic waves in a phononic crystal plate consisting of

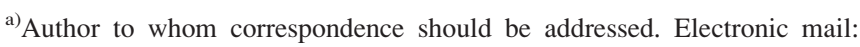
yan.pennec@univ-lille1.fr
}

piezoelectric inclusions placed periodically in an isotropic host material. ${ }^{13}$ In these papers, the origin of the modes is discussed according different resonances coupling with the diffractive waves. The ones that are currently admitted to govern the acoustic properties of the plates under normal excitation are the lattice resonances in periodic arrays, Fabry-Pérot modes of the hole cavities, localized eigenmodes, and elastic Lamb modes.

Recently, a new topic merged, turned toward the existence of simultaneous phononic and photonic band gaps. The phoXonic band gap has been demonstrated for example, in silicon crystal membranes with periodic array of air holes ${ }^{14,15}$ or in periodic arrays of silicon pillars on thin homogeneous $\mathrm{SiO}_{2}$ plate. ${ }^{16}$ The introduction of defects inside dual photonic and phononic crystals gave the opportunity to trap both phonons and photons in the same cavity for the purpose of enhancing their optomechanical interaction. ${ }^{17-19}$

Our goal is to estimate the potentiality of a phoxonic crystal plates to be an efficient tool for sensing simultaneously the light and sound velocities of liquids in a same structure. From the point of view of sensing applications, some authors have studied the normal transmission of optical waves through a plate perforated periodically with holes and measured the shift of a well-defined feature in the spectrum with the refractive index of the embedding liquid. ${ }^{20-23} \mathrm{~A}$ similar concept has been applied by Cunningham as direct biochemical assay technique $^{24}$ and further developed as label-free imaging of cell attachment with photonic crystal enhanced microscopy. ${ }^{25}$ Phononic crystals plates have recently been introduced as a new sensor platform with normal incidence of sound ${ }^{26}$ as well 
as phononic crystal sensor featuring a liquid filling air inclusions $^{27}$ or cavity defect. ${ }^{28}$ Salman et al..$^{29}$ demonstrate numerically the determination of ethanol concentration through a linear waveguide in a two dimensional phononic crystal of water cylinders in a mercury host. Oseev et al..$^{30}$ have shown the determination of gasoline properties with a phononic crystal cavity sensor. By the way, a microfluidic resonator has been opto-mechanically actuated using non-solid phases of matter. ${ }^{31}$ A theoretical investigations of a new sensor platform, the phoXonic sensor, was reported recently for in-plane waves in $2 \mathrm{D}$ infinite periodic crystal. ${ }^{32}$

In this paper, we discuss in details the novel concept of dual phononic and photonic plate for sensing the light and sound velocities of biochemical analytes, under normal incidence. The structure is composed of a silicon crystal plate drilled with air holes. The whole device is immersed in a liquid. The goal is to define a highly sensitive phoxonic sensor sensitive simultaneously to the optical and acoustic index of refraction of the fluid. Such ultra compact structure then represents a label-free, affinity-based acoustic and optical nanosensor, useful for biosensing applications.

The outline of the paper is described as follows. In Sec. II, we first define the geometrical and physical parameters of the structure before coming to the presentation of the finite difference time domain (FDTD) method used for the calculation of the photonic and phononic transmission coefficient. We investigate in Section III the phononic and photonic transmission of an incident wave launched normally to the drilled plate embedded in the liquid. Section IV is devoted to a discussion on the choice of the geometrical parameters and how they affect the trend of the well-defined features in the transmission coefficients with the acoustic and optical properties of the analyte. This study allows the design of a high sensitive phononic/photonic crystal sensor. Finally, the conclusions are drawn in Section V.

\section{GEOMETRICAL PARAMETERS AND METHOD OF CALCULATION}

We study the normal transmission through a periodically perforated silicon plate with a lattice constant, $a$, a hole radius, $r$, and a thickness $h$ (Figure 1(a)). The plate is totally immersed in a liquid. Silicon is considered as a cubic material with the elastic constants $C_{11}=16,57 \times 10^{10}\left(\mathrm{~N} / \mathrm{m}^{2}\right)$, $C_{44}=7.962 \times 10^{10}\left(\mathrm{~N} / \mathrm{m}^{2}\right), C_{12}=6.39 \times 10^{10}\left(\mathrm{~N} / \mathrm{m}^{2}\right)$, and with a mass density $\rho=2331 \mathrm{~kg} / \mathrm{m}^{3}$. From the optical point of view, silicon behaves as an isotropic medium with a refractive index $n=3.5$. The embedded medium is firstly chosen as water with acoustic wave velocity $c_{\text {water }}=1490 \mathrm{~m} / \mathrm{s}$ and optical refractive index 4/3.

All photonic and phononic transmission curves presented in the paper have been obtained with the help of homemade 3D finite difference time domain (3D-FDTD) codes. The FDTD approach has been first used extensively with success to study the propagation of electromagnetic waves through photonic band gap materials. ${ }^{33}$ Later on, the FDTD method has been extended to phononic crystals. ${ }^{34}$ This method solves either the electromagnetic or the elastic wave equations by discretizing time and space and by replacing derivatives by finite
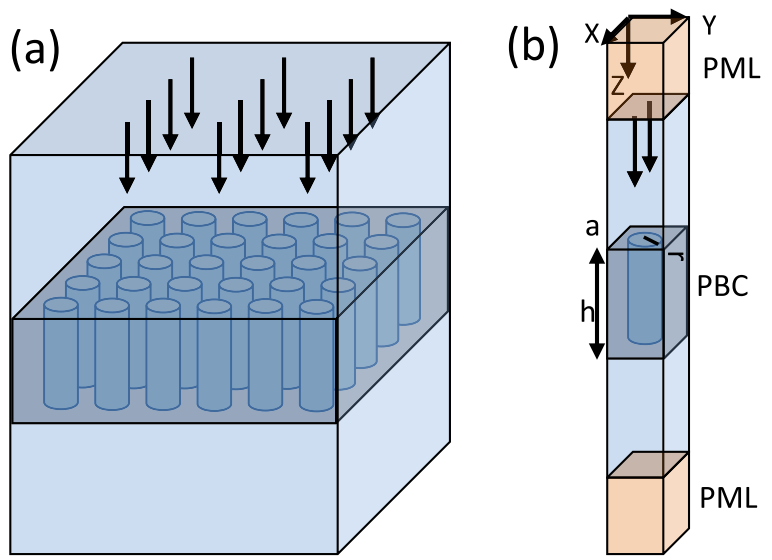

FIG. 1. (a) Three-dimensional schematic representation of a normal transmission through a silicon periodic plate embedded in a liquid. (b) Elementary unit cell used for the FDTD calculation of the photonic and phononic transmission. On each y-boundaries of the unit cell, perfect matching layers (PML) are applied, while on the $\mathrm{x}$-boundaries, periodic boundary conditions (PBC) insure the periodicity of the crystal. The phononic/photonic crystal plate of thickness $h$ has a lattice parameter $a$ and a radius $r$.

differences in the equations of motion. As compared to other numerical method such as plane wave expansion (PWE), it presents the advantage of allowing simulation of mixed phononic structures, composed of both fluids and solids.

Figure 1(b) represents the elementary unit cell used for the calculation. For instance, we build a sample in three parts along the direction of propagation y. Perfect matching layers $(\mathrm{PML})^{35}$ are imposed at the free ends of the homogeneous regions to avoid the reflective waves. The crystal is constituted of cylindrical inclusions of finite height along the $\mathrm{z}$ direction, arranged periodically on a square lattice in the $(\mathrm{x}, \mathrm{y})$ plane. The lattice is periodically infinite along $\mathrm{x}$ and $\mathrm{y}$. The input signal is launched from the left of the unit cell, perpendicularly to the plate. The transmitted signal is then recorded at the end of the second homogeneous region and integrated along the $(\mathrm{x}, \mathrm{y})$ plane. Accordingly, the space is discretized in all directions with a mesh interval of $a / 30$ in photonic and $a / 40$ in phononic for a good convergence of the calculation. The equations of motion are solved with a time integration of $t=2^{19} . d t$ in both photonic and phononic calculations. To yield the transmission coefficient, the outgoing signal, recorded as a function of time, is Fourier transformed and normalized to the Fourier transform of a signal propagating through a homogeneous liquid. The transmission curves are, in general, presented as a function of a normalized frequency $\Omega=\omega a / 2 \pi c$, where $\omega$ is the angular frequency (in s ${ }^{-1}$ ), and $c$ is either the velocity of light in vacuum for the electromagnetic waves or the transverse velocity of sound in bulk silicon.

\section{DUAL PHOTONIC AND PHONONIC TRANSMISSIONS}

\section{A. Photonic calculations}

We present Figure 2 the photonic calculations for an incident wave launched perpendicularly to the plate when the embedded medium is water (refractive index $n_{\text {water }}=1.33$ ). The transmission spectrum consists of sharp resonant features on a smooth Fabry-Pérot background. The physical origin of these 


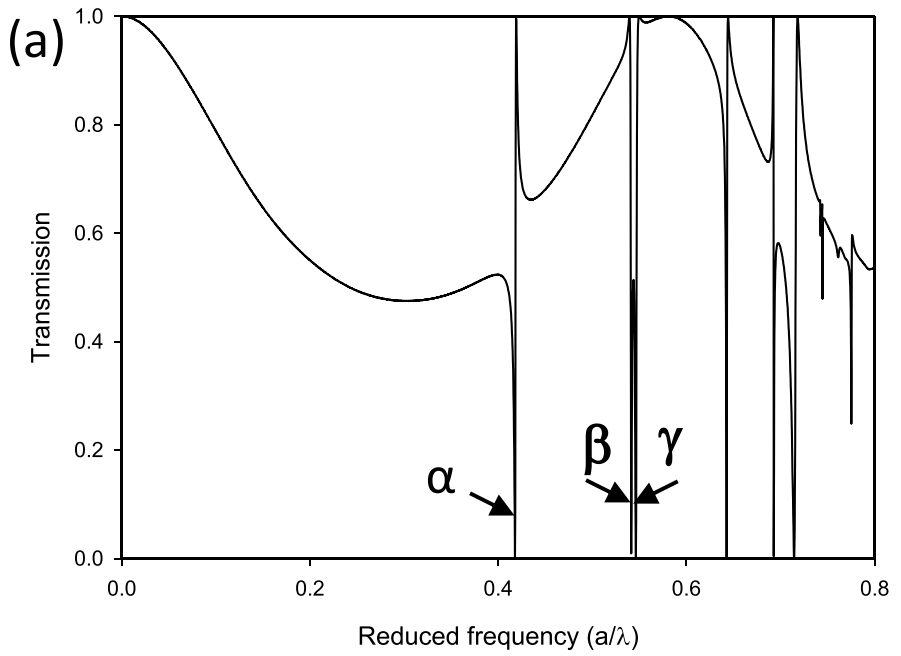

(c)

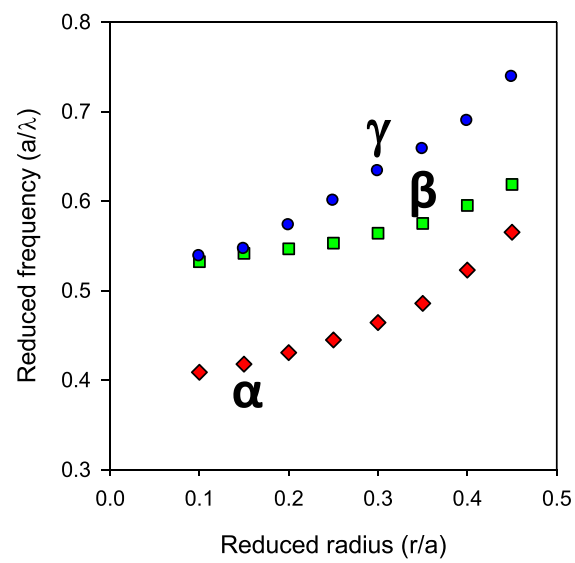

(b)
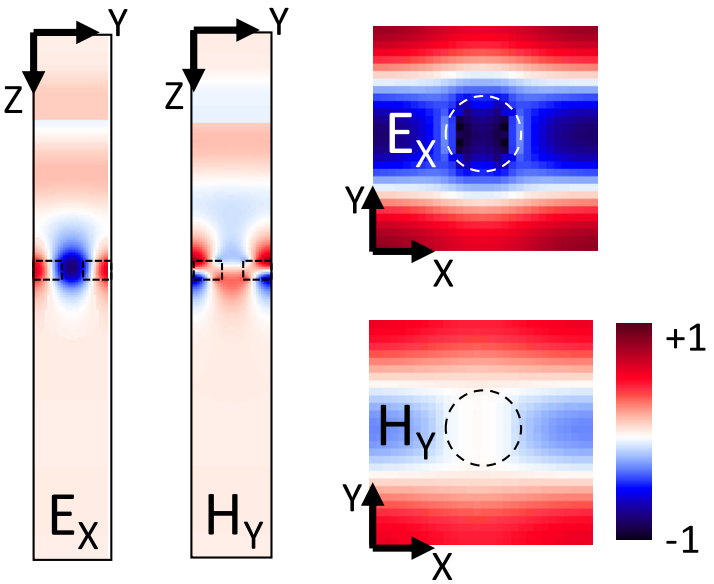

(e)

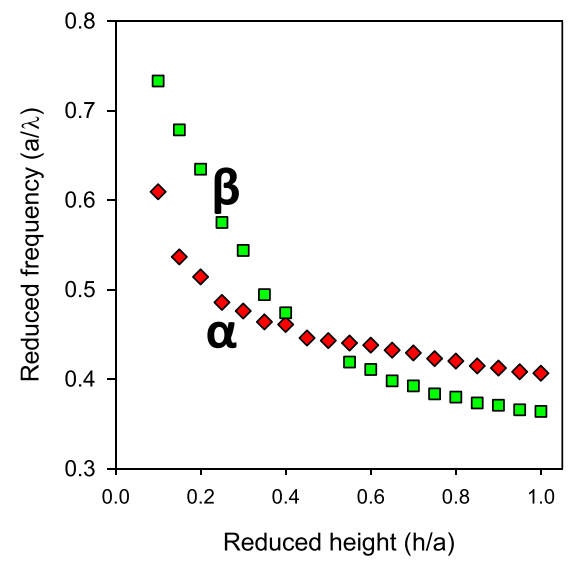

FIG. 2. (a) Photonic transmission curve for an incident wave launched along $\mathrm{z}$, normally to the plate $(h / a=0.25, r / a=0.15)$, and when the embedded medium is water. (b) Cartography of the electric and magnetic fields at the frequency of the dip $\alpha$. Evolution of the modes as a function of (c) the radius $r / a$ when $h / a=0.25$, (d) and (e) the height $h / a$ when $r / a=0.15$ and $r / a=0.35$, respectively.

resonant features is known ${ }^{3,4}$ and can be understood by considering the Bragg scattering provided by the photonic crystal lattice which folds the modes of the dielectric plate into the first Brillouin zone. This brings modes above the light line, at the $\Gamma$ point of the Brillouin zone, allowing them to be coupled to by normal light illumination.

In the purpose of this study, we note that, with the geometrical parameters fixed to $r / a=0.15$ and $h / a=0.25$, the transmission curve (Figure 2(a)) leads to a well-defined and isolated peak/dip $\alpha$ at the reduced frequency 0.42 with an asymmetric shape. The map of the electromagnetic field at the frequency of the dip $\alpha$ is presented in Figure 2(b). One can see that the electromagnetic field is strongly confined and spread over the whole plate, both inside the water hole and the silicon matrix. As reported in the literature, ${ }^{3,4}$ such a mode is referred as a guided resonance mode of the dielectric plate and comes from the excitation at $\mathrm{k} / /$ equal to zero of the symmetric modes of the periodic plate.

The peak $\alpha$, well-isolated in the transmission spectrum, presents a high quality factor due to its asymmetric shape. Such a peak is suitable for the photonic sensing purpose. In the transmission curve, the peak $\alpha$ is followed by another $\operatorname{dip}, \beta$ at $a / \lambda=0.55$. However, the variation of the transmissions frequencies as a function of the radius $r(h / a=0.25$, Figure 2(c)) shows that the second peak is the consequence of the superposition of two others, $\beta$ and $\gamma$, which gather at low radius values. The evolution of the three modes $\alpha, \beta$, and $\gamma$ has been reported as a function of the height $h$ of the plate, for, respectively, a small radius $(r / a=0.15$, Figure 2(d)) and a larger one $(r / a=0.35$, Figure 2(e)). All frequencies increase (resp. decrease) as far as the radius (resp. height) increases, in good agreement with the corresponding optical effective index variation. It remains from this parametric study that, depending on the thickness of the plate and the radius of the holes, the structure presents several sharpen and isolated peaks in the transmission spectra then offer the opportunity to be used for sensing application.

\section{B. Phononic calculations}

Figure 3(a) represents the acoustic transmission for a wave launched normally to the plate and when the embedded medium is water of density $d_{\text {water }}=1$ and acoustic wave velocity $c_{\text {water }}=1490 \mathrm{~m} / \mathrm{s}$. The transmission curve shows a well-defined asymmetric peak (A) at the reduced frequency 

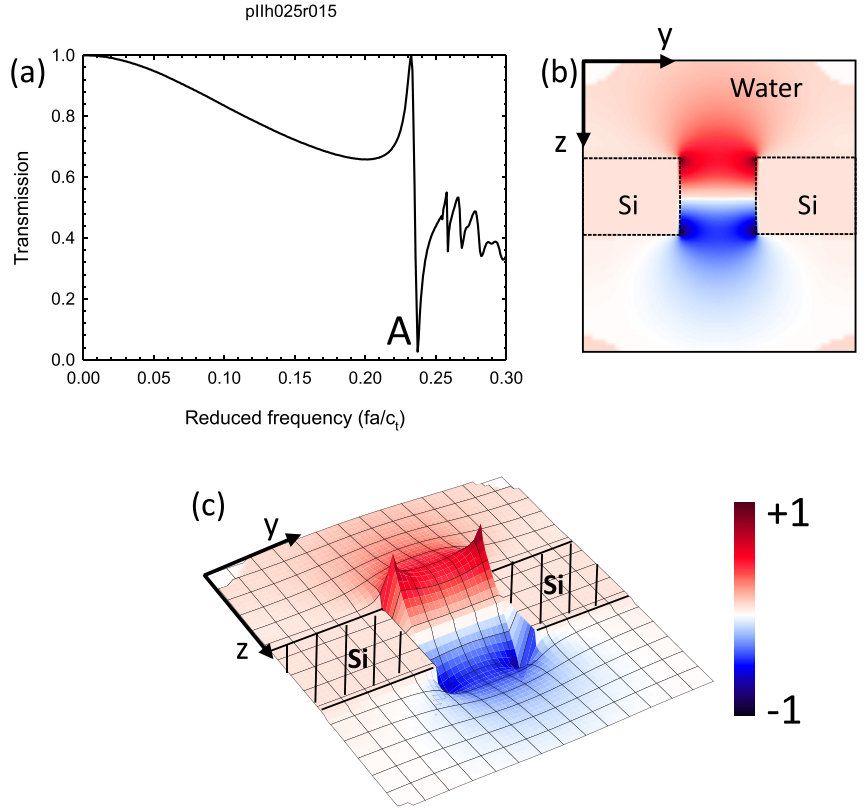

FIG. 3. (a) Phononic transmission curve for an incident wave launched along $\mathrm{z}$, normally to the plate $(h / a=0.25, r / a=0.15)$, and when the embedded medium is water. Representation of the displacement field $U_{z}$ at the frequency of mode A. 2D (b) and 3D (c) cross section in the (y, z) plane, cutting the water hole along his diameter.

0.235. This kind of peak/dip feature is known as Fano-like or hybridized modes as introduced in Ref. 36.

The origin of the asymmetric peak $\mathrm{A}$ in the transmission spectrum is discussed with respect to the component $\left|U_{z}\right|$ of the displacement field at the frequency of mode A, considering the cross section in the $(y, z)$ plane, cutting the water hole along the diameter (Figure 3(b)). The mode A is strongly localized at the four extremities of the cylindrical inclusion (dark points) before decreasing all around in the water. The Figure 3(c) shows the same calculation in 3D to highlight the decreasing of the field. In other worlds, it means that the field is localized at the circular edge of the cylindrical inclusion and is at the origin of a confined mode inside the cavity. The asymmetric peak/dip A, observed in the transmission curve calculation, is then the consequence of the coupling between the propagative incident wave and the cavity mode, leading to the Fano-shape resonance in the transmission spectrum.
The evolution of the eigenfrequency of mode A has been investigated as a function of the geometrical parameters $r$ and $h$ (Figure 4). While the frequency is almost constant when we increase the radius of the hole, it drastically falls down to the low frequencies when we increase the height of the plate. This behavior is in good agreement with the spreading of the stationary wave inside the water cylinder axis, leading to a larger wavelength of excitation, then to a lower frequency. The sensitivity of the eigen frequency as a function of the height of the plate opens the way to a tunability of the driven frequency which represents a welcome property for the sensing purpose.

\section{PHONONIC/PHOTONIC SENSOR}

A high efficient phononic/photonic sensor should present a transmission reply which displays very sensitive features to the acoustic and optical velocity of the infiltrating analyte. The preliminary condition is to get isolated features (peaks or dips) in order to allow the sensing of the probed parameter on a sufficiently broad frequency range. One can see from the evolution of the asymmetric phononic peak A (Figure 4) that no conditions are required on the geometrical parameters to respect this condition as far as the thickness is lower than $h / a=1$. It means that the limitation on the structure should come from the photonic side. As seen in Figure 2(c), considering the peak $\alpha$, all radii can be chosen for the average thickness $h / a=0.25$. In the following, we first start with a small $(r / a=0.15)$ and large radius $(r / a=0.35)$ with $h / a=0.25$ and proceed to the estimation of the sensor efficiency in both case.

The efficiency of the sensor has been tested by changing the physical parameters of the embedded analyte, namely, the acoustic and optical velocities. In phononic and photonic, we have considered a same set of mixtures made of ionic liquid 1-Methyl-3-octylimidazolium Chloride with Methanol at different molar ratio $x$. The density $\rho_{\text {liq }}$, speed of sound $v_{\text {liq }}$, and refractive indexes $n_{l i q}$ of the analyte, obtained from Ref. 37, are reported in Table I as a function of $x$.

Figures 5(a) and 5(b) show the behavior of the phononic and photonic transmission coefficients considering, respectively, the acoustic velocity and refractive index of the analyte. In both cases, the two asymmetric peaks, $\mathrm{A}$ in phononic
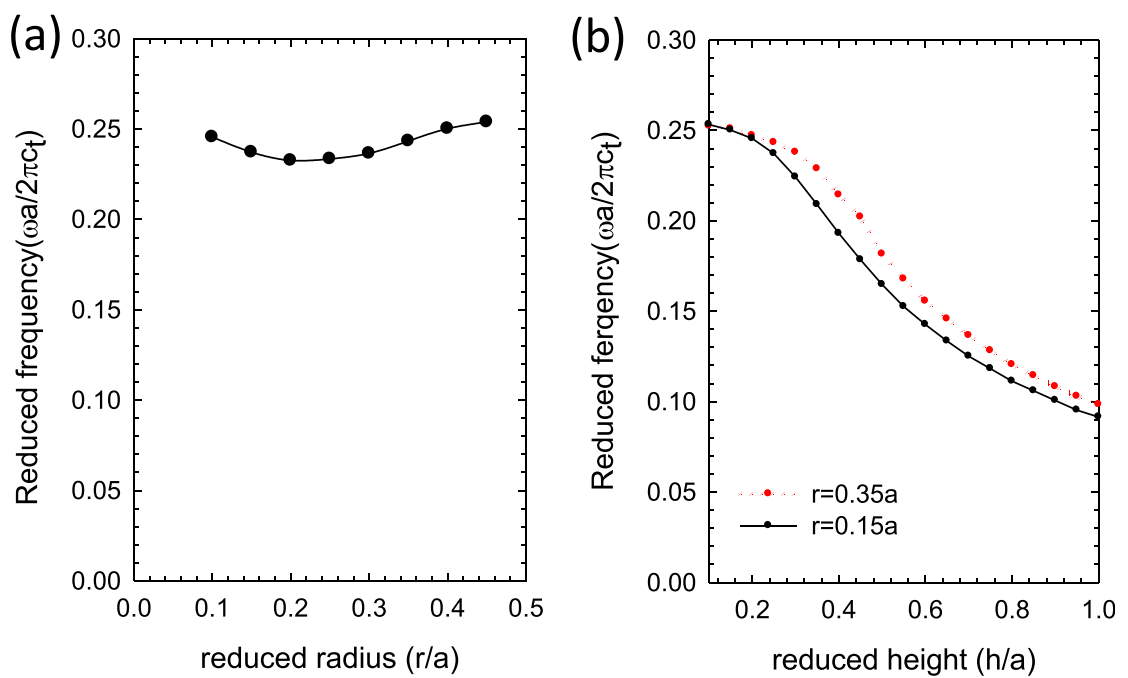

FIG. 4. Evolution of the eigenfrequency of mode $\mathrm{A}$ as a function of (a) the radius $r / a$ when $h / a=0.25$ and (b) the height $\mathrm{h} / \mathrm{a}$ when $r / a=0.15$ and $r / a=0.35$, respectively. 
TABLE I. Density $\rho_{l i q}$ and speed of sound $c_{l i q}$ and refractive index $n_{l i q}$ of ionic liquid 1-Methyl-3-octylimidazolium Chloride in Methanol for different molar ratio $x .^{37}$

\begin{tabular}{lrcc}
\hline \hline Molar ratio, $x$ & $\begin{array}{c}\text { Density } \\
\rho_{\text {liq }}\left(\mathrm{kg} / \mathrm{m}^{3}\right)\end{array}$ & $\begin{array}{c}\text { Longitudinal sound } \\
\text { velocity }\left(c_{\text {liq }}\right)(\mathrm{m} / \mathrm{s})\end{array}$ & $\begin{array}{c}\text { Refractive } \\
\text { index }\left(n_{\text {liq }}\right)\end{array}$ \\
\hline 0.0000 & 1008.82 & 1721 & 1.5105 \\
0.5589 & 973.32 & 1526 & 1.4769 \\
0.7536 & 939.25 & 1447 & 1.4478 \\
0.8713 & 897.37 & 1348 & 1.4127 \\
0.9436 & 850.42 & 1237 & 1.3757 \\
1.000 & 786.64 & 1103 & 1.3268 \\
\hline \hline
\end{tabular}

and $\alpha$ in photonic, shift in frequency with the different liquids. In photonic, when the refractive index increases, the reduced frequency " $\alpha$ " decreases (Figure 5(b)). In phononic, when the longitudinal velocity decreases, which corresponds to an increase of the acoustic refractive index, the reduced frequency of dip A decreases as well. As a conclusion, the phononic and photonic behavior is the same: when the refractive index increases, the eigenmode wavelength is red-shifted.

The sensitivity of the sensor has been estimated in the telecommunication range which is reached when the period of the crystal is taken equal to $640 \mathrm{~nm}$. With the objective of the dual sensor, the choice of the geometrical parameters, especially the pitch " $a$," is done in the way to get the properties in a frequency range that can be easily captured in both acoustic and optical spectra. The only way to respect this condition is to choose a lattice parameter at the submicron scale that leads to $\mathrm{GHz}$ frequency in phononic and $\mu \mathrm{m}$ wavelength in photonic. With this lattice parameter, and when the embedded medium is filled with water, the wavelength of modes $\alpha$ occurs at $1533 \mathrm{~nm}$ while the frequency of mode $\mathrm{A}$ is at $2.13 \mathrm{GHz}$.

We report Figure 6(a) the evolution of the phononic frequencies as a function of the longitudinal velocity of the analyte and in Figure 6(b) the evolution of the photonic wavelengths as a function of the refractive index. In both case, the sensitivity corresponds to the slope of the curve which is linear and defined by, in phononic

$$
S_{\text {phononic }}^{i}=\frac{\Delta f_{i}}{\Delta c_{\text {liq }}}\left(\mathrm{MHz} / \mathrm{ms}^{-1}\right),
$$

and in phononic

$$
S_{\text {photonic }}^{j}=\frac{\Delta \lambda_{j}}{\Delta n_{\text {liq }}}(\mathrm{nm} / \mathrm{RIU}),
$$

where RIU is the Refractive Index Unit.

Another way widely used in photonic ${ }^{38}$ to characterize the sensing capabilities is given by the calculation of the figure of merit (FoM), which is the way to take into account the thickness of the peaks through the quality factor

$$
F o M_{\text {photonic }}^{j}=\frac{S_{j} \times Q_{j}}{\lambda_{j}}\left(\mathrm{RIU}^{-1}\right)
$$

where $Q_{j}=\frac{\lambda_{j}}{\Delta \lambda_{j}}$ is the photonic quality factor of mode $\mathrm{j}$.

In parallel, one can define an equivalent figure of merit in phononic as follows:

$$
F_{o} M_{\text {phononic }}^{i}=\frac{S_{i} \times Q_{i}}{f_{i}}(\mathrm{~m} / \mathrm{s})^{-1},
$$

where $Q_{i}=\frac{f_{i}}{\Delta f_{i}}$ is the phononic quality factor of mode $\mathrm{i}$. (a)
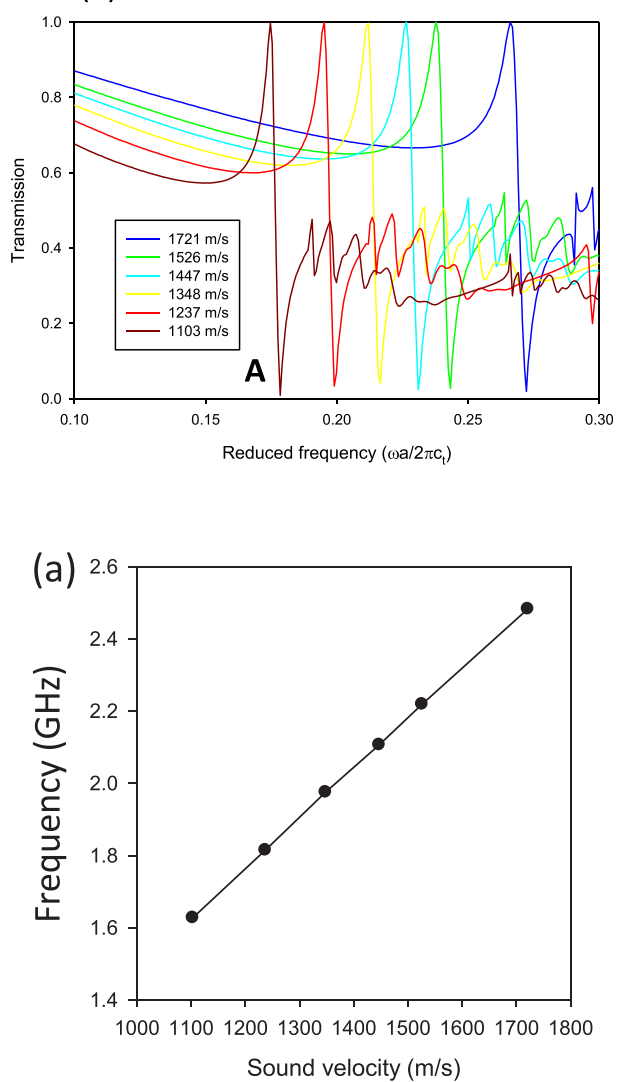

(b)
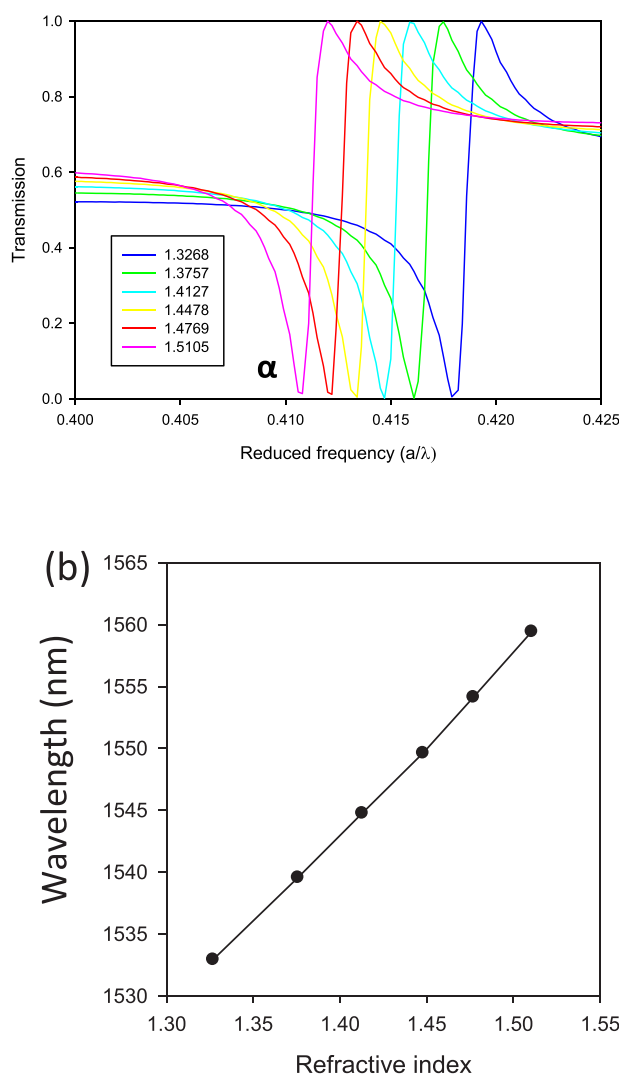

FIG. 5. (a) Evolution of the phononic transmission spectrum as a function of the acoustic velocities of the embedded medium. The inset indicates the different values of the longitudinal velocities of the probed analyte. (b) Evolution of the photonic transmission spectrum as a function of the refractive index of the liquid $(h / a=0.25$ and $r / a=0.15)$. The inset indicates the different values of the refractive index of the probed analyte.
FIG. 6. (a) Evolution of the phononic frequency of modes A as a function of the acoustic velocity of the analyte. (b) Evolution of the photonic wavelength of mode $\alpha$ as a function of the refractive index of the analyte. 
TABLE II. Phononic and photonic sensitivities $(S)$ and figure of merit $(F o M)$ for $h / a=0.25$.

\begin{tabular}{|c|c|c|c|c|c|c|c|}
\hline h/a & $\mathrm{r} / \mathrm{a}$ & $S_{\text {phon }}^{A}\left(\mathrm{MHz} / \mathrm{ms}^{-1}\right)$ & FoM $M_{\text {phon }}^{A}\left(\mathrm{~ms}^{-1}\right)$ & $S_{\text {phot }}^{\alpha}(\mathrm{nm} / \mathrm{RIU})$ & FoM $M_{p h o t}^{\alpha}$ & $S_{\text {phot }}^{\beta}(\mathrm{nm} / \mathrm{RIU})$ & FoM ${ }_{\text {phot }}^{\beta}$ \\
\hline 0.25 & 0.15 & 1.35 & 67 & 150 & 82 & l & \\
\hline 0.25 & 0.35 & 1.42 & 70 & 263 & 144 & 400 & 67 \\
\hline 0.50 & 0.15 & 0.78 & 38 & 101 & 55 & I & \\
\hline 0.50 & 0.35 & 0.8 & 39 & 188 & 103 & / & \\
\hline
\end{tabular}

Table II summarizes the results of the sensitivities and FoM calculated for the radii $r / a=0.15$ and $r / a=0.35$, with a thickness of the plate of $h / a=0.25$. One can see that the phononic sensitivity and FoM are almost the same while in photonic the mode $\alpha$ presents higher sensitivity and FoM for the larger radius. We also report the calculations for a thicker plate $(h / a=0.5)$. In both cases, phononic and photonic sensibilities and FoM decrease. One can conclude that the higher efficiency of the dual sensor is obtained for thin plate and large radii (hacked line in Table II).

\section{CONCLUSIONS}

This contribution shows the dual existence of optical and acoustic enhanced resonances inside silicon phononic and photonic (phoxonic) crystal plate under normal incidence of light and sound. These resonances lead to asymmetric peaks with Fano-like shape in the phononic and photonic transmission spectra for which the origin is totally different. In photonic, the asymmetric resonance comes from the coupling of the incident wave with a guided resonant mode of the dielectric plate. In phononic, the incident wave couples with a mode confined in the holes and due to the water/silicon edges deformation of the cylindrical inclusion. The geometrical parameters of the phononic/photonic crystal have been adapted to define structures in which the peaks of transmission are well-defined and sufficiently isolated from each others. We then showed that this structure can be used as a sensor for separately sense the sound and light velocity of unknown liquids with a high sensitivity to the acoustic/optical parameters of the infiltrating liquid. We demonstrate that the dual phononic and photonic sensor behaves in the same way: an increase of the refractive index velocity leads to a red shift of the eigenmode. We showed that a higher sensitivity and FoM are obtained for larger radius and thinner plate. We then proposed a new class of phononic/photonic sensors which have the possibility to probe light and sound velocity of unknown liquid through a same structure with high sensitivity. Future work will deal with finding the optimal wavelength/frequency scale. Optical sensors including photonic crystal sensors typically work at a few $\mu \mathrm{m}$ whereas only a few acoustic sensors apply the respective $\mathrm{GHz}$ frequencies. ${ }^{39}$ When going for liquid sensing, a major challenge must be considered which may introduce a frequency limit, viscous damping. Shear waves strongly decay within a few wavelength distance, whereas dissipation of longitudinal waves depends on bulk $(\mu)$, and shear viscosity $(\eta)$, the ratio of specific heats $(\gamma)$, thermal conductivity $(\tau)$, and density $(\rho)$ of the fluid. Experimental data of simple liquids are available for frequencies up to $200 \mathrm{MHz}$. Recently, Holmes et al. ${ }^{40}$ have analyzed Millipore water by an acoustic spectroscopy and have proven an expression for the dissipation term in the longitudinal wave number $(\alpha)$

$$
\alpha=\frac{\omega^{2}}{2 \rho c^{3}}\left[\mu+\frac{4}{3} \eta+\frac{(\gamma-1) \tau}{c_{p}}\right] .
$$

Finally, such structures can also open the way to enhance the phonon-photon interactions with the simultaneous confinement of the phononic and photonic waves. Through the confinement and the interaction between optical and acoustic waves inside mixed phoXonic crystals, we expect the enhancement of the properties of dual sensors.

${ }^{1}$ T. W. Ebbesen, H. J. Lezec, H. F. Ghaemi, T. Thio, and P. A. Wolff, Nature 391, 667 (1998).

${ }^{2}$ C. Genet and T. W. Ebbesen, Nature 445, 39 (2007).

${ }^{3}$ S. Fan and J. D. Joannopoulos, Phys. Rev. B 65, 235112 (2002).

${ }^{4}$ K. B. Crozier, V. Lousse, O. Kilic, S. Kim, S. Fan, and O. Solgaard, Phys. Rev. B 73, 115126 (2006).

${ }^{5}$ S. Fan, W. Suh, and J. D. Joannopoulos, J. Opt. Soc. Am. A 20, 569 (2003). ${ }^{6}$ X. Zhang, Phys. Rev. B 71, 241102 (2005).

${ }^{7}$ M.-H. Lu, X.-K. Liu, L. Feng, J. Li, C.-P. Huang, Y.-F. Chen, Y.-Y. Zhu, S.-N. Zhu, and N.-B. Ming, Phys. Rev. Lett. 99, 174301 (2007).

${ }^{8}$ H. Estrada, P. Candelas, F. Belmar, A. Uris, F. J. Garcia de Abajo, and F. Meseguer, Phys. Rev. B 85, 174301 (2012).

${ }^{9}$ J. Christensen, L. Martin-Moreno, and F. J. Garcia-Vidal, Phys. Rev. Lett. 101, 014301 (2008).

${ }^{10}$ H. Estrada, F. J. Garcia de Abajo, P. Candelas, A. Uris, F. Belmar, and F. Meseguer, Phys. Rev. Lett. 102, 144301 (2009).

${ }^{11}$ Y. Pennec, B. D. Rouhani, H. Larabi, A. Akjouj, and G. Leveque, New J. Phys. 14, 073039 (2012).

${ }^{12}$ R. P. Moiseyenko, Y. Pennec, R. Marchal, B. Bonello, and B. DjafariRouhani, Phys. Rev. B 90(13), 134307 (2014).

${ }^{13}$ A. Khelif, B. Aoubiza, S. Mohammadi, A. Adibi, and V. Laude, Phys. Rev. E 74, 046610 (2006).

${ }^{14}$ S. Mohammadi, A. A. Eftekhar, A. Khelif, and A. Adibi, Opt. Express 18, 9164 (2010).

${ }^{15}$ Y. Pennec, B. Djafari-Rouhani, E. H. El Boudouti, C. Li, Y. El Hassouani, J. O. Vasseur, N. Papanikolaou, S. Benchabane, V. Laude, and A. Martinez, Opt. Express 18, 14301 (2010).

${ }^{16}$ Y. El Hassouani, C. Li, Y. Pennec, E. H. El Boudouti, H. Larabi, A. Akjouj, O. Bou Matar, V. Laude, N. Papanikolaou, A. Martinez, and B. D. Rouhani, Phys. Rev. B 82, 155405 (2010).

${ }^{17}$ J. Gomis-Bresco, D. Navarro-Urrios, M. Oudich, S. El-Jallal, A. Griol, D. Puerto, E. Chavez, Y. Pennec, B. Djafari-Rouhani, F. Alzina, A. Martínez, and C. M. S. Torres, Nat. Commun. 5, 4452 (2014).

${ }^{18}$ S. El-Jallal, M. Oudich, Y. Pennec, B. Djafari-Rouhani, V. Laude, J. C. Beugnot, A. Martinez, J. M. Escalante, and A. Makhoute, Phys. Rev. B 88, 205410 (2013).

${ }^{19}$ T.-X. Ma, Y.-S. Wang, and C. Zhang, Opt. Commun. 312, 68 (2014).

${ }^{20}$ M. Huang, A. A. Yanik, T.-Y. Chang, and H. Altug, Opt. Express 17, 24224 (2009).

${ }^{21}$ M. El Beheiry, V. Liu, S. Fan, and O. Levi, Opt. Express 18, 22702 (2010).

${ }^{22}$ C. Nicolaou, W. T. Lau, R. Gad, H. Akhavan, R. Schilling, and O. Levi, Opt. Express 21, 31698 (2013). 
${ }^{23}$ R. Gordon, D. Sinton, K. L. Kavanagh, and A. G. Brolo, Acc. Chem. Res. 41, 1049 (2008).

${ }^{24}$ B. Cunningham, P. Li, B. Lin, and J. Pepper, Sens. Actuators, B 81, 316 (2002).

${ }^{25}$ E. A. Lidstone, V. Chaudhery, A. Kohl, V. Chan, T. Wolf-Jensen, L. B. Schook, R. Bashir, and B. T. Cunningham, Analyst 136, 3608 (2011).

${ }^{26}$ M. Zubtsov, R. Lucklum, M. Ke, A. Oseev, R. Grundmann, B. Henning, and U. Hempel, Sens. Actuators, A 186, 118 (2012).

${ }^{27}$ A. Sato, Y. Pennec, T. Yanagishita, H. Masuda, W. Knoll, B. DjafariRouhani, and G. Fytas, New J. Phys. 14, 113032 (2012).

${ }^{28}$ R. Lucklum, M. Ke, and M. Zubtsov, Sens. Actuators, B 171-172, 271 (2012).

${ }^{29}$ A. Salman, O. A. Kaya, and A. Cicek, Sens. Actuators, A 208, 50 (2014).

${ }^{30}$ A. Oseev, R. Lucklum, and M. Zubtsov, Sens. Actuators, B 189, 208 (2013).

${ }^{31}$ K. H. Kim, G. Bahl, W. Lee, J. Liu, M. Tomes, X. Fan, and T. Carmon, Light: Sci. Appl. 2, e110 (2013).
${ }^{32}$ S. Amoudache, Y. Pennec, B. Djafari-Rouhani, A. Khater, R. Lucklum, and R. Tigrine, J. Appl. Phys. 115, 134503 (2014).

${ }^{33}$ A. Taflove, The Finite Difference Time Domain Method (Artech, Boston, 1998).

${ }^{34} \mathrm{Ph}$. Lambin, A. Khelif, J. O. Vasseur, L. Dobrzynski, and B. DjafariRouhani, Phys. Rev. E 63, 066605 (2001).

${ }^{35}$ J. P. Berenger, J. Comput. Phys. 114, 185 (1994).

${ }^{36}$ R. Sainidou, N. Stefanou, and A. Modinos, Phys. Rev. B 66, 212301 (2002).

${ }^{37}$ E. J. Gonzalez, L. Alonso, and A. A. Dominguez, J. Chem. Eng. Data 51, 1446-1452 (2006).

${ }^{38}$ K. M. Mayer and J. H. Hafner, Chem. Rev. 111, 3828 (2011).

${ }^{39}$ T. Lalinský, I. Rýger, G. Vanko, M. Tomášk, I. Kosti, Š. Hašcík, and M. Vallo, Proc. Eng. 5, 152 (2010).

${ }^{40}$ M. J. Holmes, N. G. Parker, and W. J. W. Povey, J. Phys.: Conf. Ser. 269, 012011 (2011). 